PROC. OF JSCE,

NO. 219, NOV. 1973

\title{
DEVICE OF CONDENSER-TYPE SOIL STRAIN METER
}

\author{
By Koichi AKAI* and Masayuki HORI**
}

\section{INTRODUCTION}

It is of great importance in the field of soil mechanics to measure exactly local strains as well as local stresses in soil without any disturbance. Since soil stress gauge instrumentations have been developed so much, it is fairly easy to measure stresses in natural state of the ground. On the other hand, an appropriate instrumentation to measure local strains in soil has not been established in this country. Especially, it has been impossible to measure local dynamic strains in soil.

The authors devised the condenser-type soil strain meter in order to measure the one-dimensional soil deformations during stress wave propagation. ${ }^{1)}$ This type of strain meter has been used in the air but has not been used in soil as a dielectric substance. The advantages of this type of strain meter are as follows; ${ }^{2}$

(1) able to measure such a large strain that cannot be measured by strain gages,

(2) able to measure strains as well as displacement and vibrating amplitudes as it is operated without physical connection between two electrode plates, and

(3) the construction is very simple and inexpensive.

However, this meter has also disadvantages as follows;

(1) since radio frequency is used in this meter, it is influenced by a man approaching or things without shielding,

(2) it is very difficult to calibrate the relationship between the spacing of two electrode plates in soil and change in capacity, and

(3) we have a chance to disturb soil condition in burying two electrode plates in a sample.

The authors remove the above disadvantages

* Dr. Eng., Professor of Civil Engineering, Kyoto University

** M.S.C.E., Doctorial Course Student, Kyoto University by using the needles instead of the plates as the condenser constructed in the soil. One-dimensional microscopic strains of soil can be measured simply and exactly by means of this type of soil strain meter.

\section{PREVIOUS DEVICES OF SOIL STRAIN MEASUREMENT}

In U.S.A., soil strain gage instrumentation has been developed by IITRI to measure transient soil deformations in both laboratory and field applications. This strain sensor consists of two flatcoil disks which are embedded in soil in near parallel and concentric orientation without physical connection between them. The remainder of the gage hardware consists of a second set of coil disks, identical to those used as the strain sensor, and specially designed electronic driving, amplifying, balancing, and recording circuitly. Soil deformations are measured by the resulting changes in the spacing of embedded coils which are sensed as changes in the mutual inductance of the coils. ${ }^{3}$ ) This strain sensor is used so many kinds of soil testing, for example, tri-axial compression test, ${ }^{3)}$ wave propagation studies in soil4),5) and so on. However, this strain sensor have disadvantages as follows;

(1) since the size of the coil disk is relatively large (laboratory gage: $3 / 4$ inch in diameter and $1 / 16$ inch in thickness, field gage: 4 inches in diameter and $1 / 4$ inch in thick. ness), soil sample seems to be considerably disturbed in embedding the coil disks, and

(2) the equipment is expensive.

Nishigaki $i^{6)}$ emphasizes that the measurements of microscopic strain are very important to obtain the elastic constants of soils and tries to paste a strain gage on the side of soil sample of unconfined compression test. By this procedure, he obtains Young's modulus of clay in microscopic strain level. This procedure is very available with relatively stiff clay such as diluvial clay but unavailable with sandy soils or relatively soft clay 
such as alluvial one.

\section{PRINCIPLE OF OPERATION IN CON- DENSER-TYPE SOIL STRAIN METER}

\subsection{Apparatus}

In the device of this condenser-type soil strain meter, 'Infinitesimal Displacement Meter MD-31' (Iwasaki Communication Apparatus Co., Ltd.), is used. This meter, as shown in Photo. 1, consists of a meter MD-31, a probe MDG-643 and a condenser embedded in soil. A main variable condenser $(100 \mathrm{pF})$, a fine variable condenser $(20 \mathrm{pF})$ which is installed by the authors are contained in the probe MDG-643. The size, shape and material of the electrode plates as a condenser embedded in soil are optional. In the previous paper, ${ }^{1)}$ the authors used the rectangular thin copper plates $(2.5 \mathrm{~cm} \times 1.5 \mathrm{~cm})$ with coated by enamel (see Photo. 2) so as to use even in wet soils. In this paper, however, two needles $(6 \mathrm{~cm}$ in length and $0.5 \mathrm{~mm}$ in diameter) with emveloped by a vinyl tube, as shown in Photo. 3 , are used as two electrodes in order to decrease

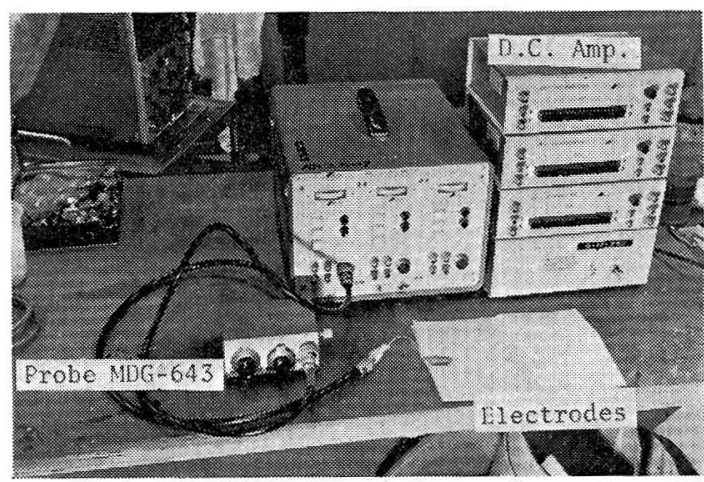

Photo. 1 Apparatus of the condenser-type soil strain meter.

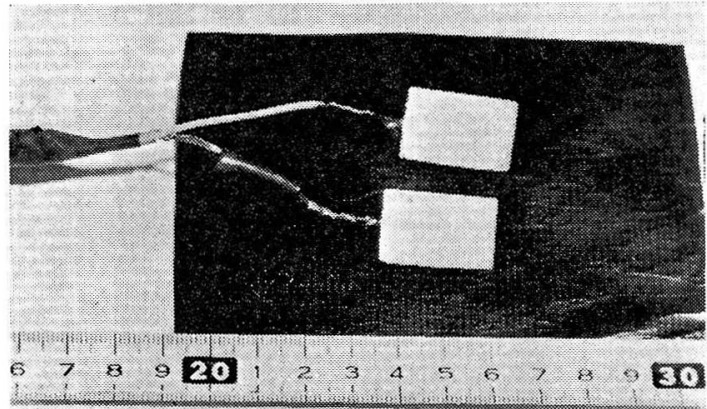

Photo. 2 The rectangular thin electrode plates with coated by enamel.

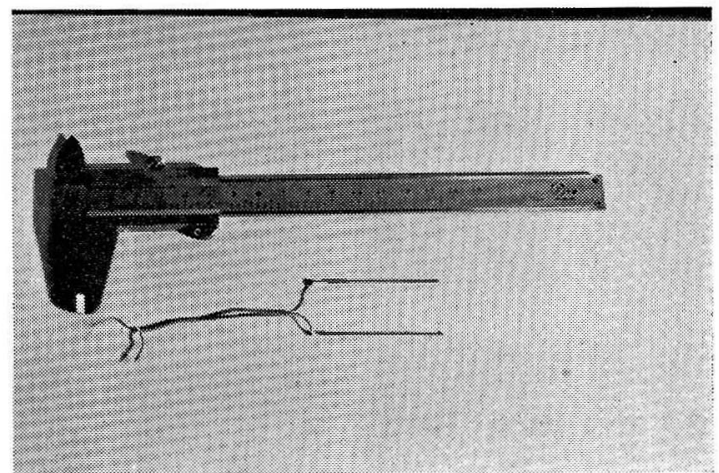

Photo. 3 The electrode needles with emveloped by a vinyl tube.

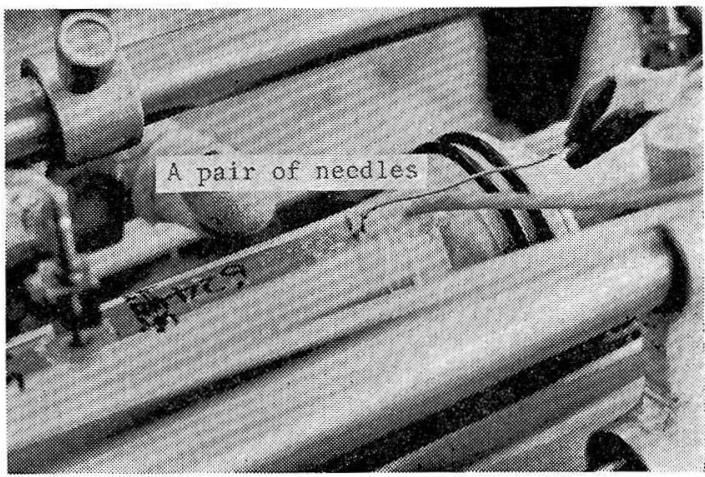

Photo. 4 The electrode needles embedded in soil sample.

disturbance of soil sample in embedding into soil. In experiments, as shown in Photo. 4, these two needles are embedded in parallel in soil. Soil deformations are measured by the resulting changes in the spacing of embedded two needles which are sensed as changes in the electrostatic capacity of them.

\subsection{Principle of Operation}

For simplicity, consider two flat rectangular electrode plates but not two needles to explain the principle of operation in this type of soil strain meter. When two conductors are placed in parallel as shown in Fig. 1, the resulting

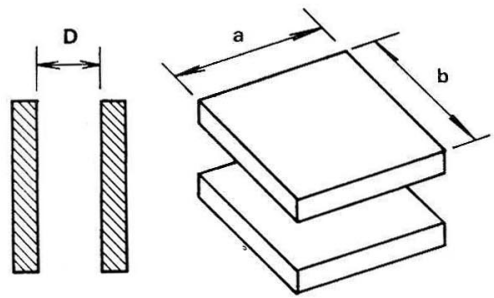

Fig. 1 Two conductors in parallel. 
electrostatic capacity is given by

$$
\begin{aligned}
C=\frac{A \varepsilon_{0} \varepsilon_{s}}{D} & =8.885 \times 10^{-12} \times \frac{A \varepsilon_{s}}{D} \\
& =8.885 \times \frac{A \varepsilon_{s}}{D}
\end{aligned}
$$

where, $A$ denotes the cross sectional area $(a \times b$ $\left.\left[\mathrm{m}^{2}\right]\right)$ of the conductors, $D$ the spacing of two conductors $[\mathrm{m}], \varepsilon_{0}$ the dielectric constant and $\varepsilon_{s}$ the specific dielectric constant. Eq. (1) implies that if $\varepsilon_{s}$ is assumed to be constant through testing, the variation in the spacing $\Delta D$ of two conductors results in the change in capacity $\triangle C$ :

$$
\Delta C=\frac{\partial C}{\partial D}=-8.885 \times \frac{A \varepsilon_{s}}{D^{2}} \Delta D
$$

The strain meter consists of the bridge circuit indicated in Fig. 2, where $C_{3}$ and $C_{4}$ denote the

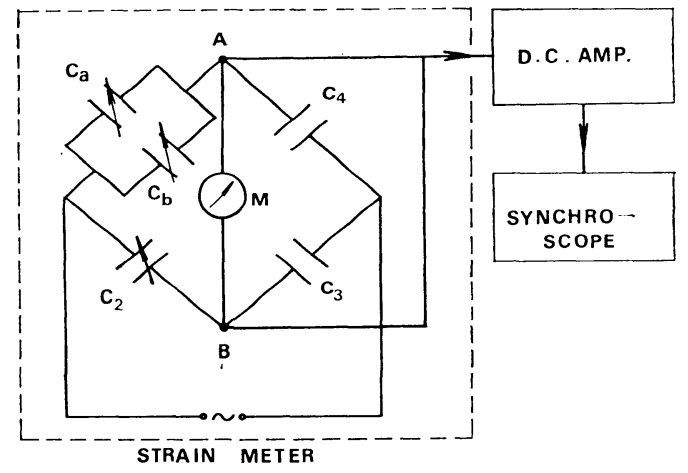

Fig. 2 Bridge circuit in the strain meter.

fixed condensers and has also equal capacity, $C_{2}$ the variable condenser for balance, $C_{a}$ the measuring condenser embedded in soil and $C_{b}$ the variable subcondenser which is used when $C_{a}$ is less than $20 \mathrm{pF}$. If $C_{a}+C_{b}$ is replaced by $C_{1}$, a balance condition for this bridge circuit due to these four condensers $\left(C_{1}, C_{2}, C_{3}\right.$ and $\left.C_{4}\right)$ is given by

$$
\frac{C_{1}}{C_{2}}=\frac{C_{3}}{C_{4}}=1
$$

i.e., if $C_{2}$ is so controlled that it is equal to $C_{1}$ for measurement, no electric current occurs in the meter $M$. When $C_{1}$ changes, however, a potential difference occurs between the point $A$ and $\mathrm{B}$ shown in Fig. 2, and an electric current flows in meter $M$.

\section{CALIBRATION}

A relationship between a change in spacing of two electrode plates and a resulting potential difference should be obtained by a calibration test.
The previous manner of calibration ${ }^{1)}$ was that the soil sample was packed in a ring made of vinylchloride of which inside diameter and height were $5.2 \mathrm{~cm}$ and $2.0 \mathrm{~cm}$, respectively. Two electrode plates were put on the upper and the lower surface of the packed soil sample in concentric orientation so that the initial spacing between two plates was about $2.0 \mathrm{~cm}$. The initial spacing of them was accurately measured by the travelling microscope. After setting up the apparatus and balancing the bridge circuit in the prove MDG643 , soil sample was compressed by means of unconfined compression apparatus and, at the same time, the resulting potential difference was measured by means of a synchroscope. This manner of calibration test, however, was rather primitive so that the measurements of soil strain was relatively inaccurately. The authors found that such a calibration test was needless.

When a pair of electrode needles is embedded in the soil sample in parallel the electrostatic capacity stored in this condenser can be expressed by the expression same as Eq. (1) to the rectangular electrode plates,

$$
C_{0}=\frac{a}{D_{0}} \quad[\mathrm{pF}]
$$

where, $C_{0}$ denotes the initial capacity stored in the condenser $[\mathrm{pF}], D_{0}$ the initial spacing of the two needles [m] and $a$ the constant which is determined by the size and shape of the electrode needles. When the soil sample deforms and $\Delta D$ changes in the spacing of the two needles, the corresponding change of the capacity $\triangle C$ is expressed by the following equation if $\Delta D$ is very small,

$$
\begin{aligned}
& \Delta C=-\frac{a}{D_{0}{ }^{2}} \Delta D=-\frac{a}{D_{0}} \frac{\Delta D}{D_{0}} \\
& =-C_{0} \frac{\Delta D}{D_{0}}
\end{aligned}
$$

Since $\frac{\Delta D}{D_{0}}$ is a quantity of soil strain, Eq. (5) means that the corresponding change in capacity $\triangle C$ with the change in spacing $\Delta D$ is proportional to the soil strain. Namely, if the initial capacity $C_{0}$ is known, the soil strain is calculated from Eq. (5). The initial capacity $C_{0}$ is easily obtained by the following equation;

$$
C_{0}=C_{A}-C_{B} \quad[\mathrm{pF}]
$$

where, $C_{A}$ denotes the capacity with the condenser embedded in the soil and $C_{B}$ the capacity without it.

When we observed the strain record by means of a synchroscope or an electromagnetic oscillo- 


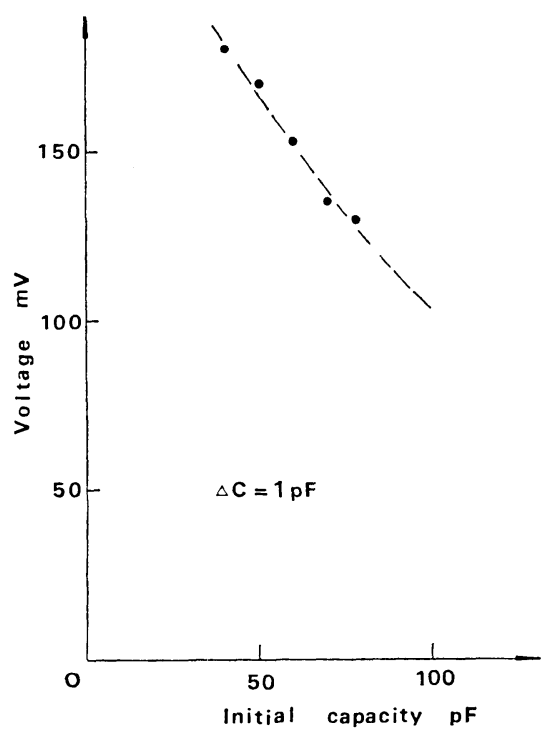

Fig. 3 Typical results of the relationship between the initial capacity and the corresponding voltage.

graph, we must obtain the relationship between the change in capacity $\Delta C$ and the corresponding voltage $\Delta V$ from calibration test. This relationship depends on the initial capacity. Typical test results are shown in Fig. 3 as $\Delta C=1 \mathrm{pF}$. If the relationship between $\Delta C$ and $\Delta V$ at a certain initial capacity $C_{0}$ is expressed by the following equation;

$$
\Delta V=\frac{1}{k} \Delta C \quad[\mathrm{mV}]
$$

where, $k$ denotes a constant, then the strain $\varepsilon$ can be calculated from,

$$
\varepsilon=\frac{\Delta D}{D_{0}}=-\frac{\Delta C}{C_{0}}=-\frac{k}{C_{0}} \Delta V
$$

The authors have confirmed that the linearity between strain $\varepsilon$ and voltage $\Delta C$ exists in the range of strain less than $\left.2 \% .{ }^{7}\right)$

\section{APPLICATIONS}

\subsection{One-Dimensional Stress Wave Propagation Test}

The soil strain meter is used in the one-dimensional stress wave propagation test in clay in order to obtain the stress-strain relationships during rod wave propagation. ${ }^{8}$ ) The rod of clay is $135 \mathrm{~cm}$ in length and $7.5 \mathrm{~cm}$ in diameter which is consolidated at confining pressure $p=1.06$ and $0.54 \mathrm{~kg} / \mathrm{cm}^{2}$ in the special acryl cell designed by the authors. ${ }^{8}$ ) Then the cell is relieved and a pair of needles as a strain meter is embedded in parallel with the initial spacing of about $1 \mathrm{~cm}$. At the one end of the rod, the pulsative stress is applied by means of the shock tube. When the applied dynamic stress is propagating in the rod as a wave, the stress and the strain are at the same time measured by the soil stress gauge embedded in the sample and the strain meter, respectively. Figs. 4 (a) and (b) show the typical records of the stress and the strain forms recorded by a synchroscope. The observation depth, and the ratio of the proportional constant $k$ between the differential voltage and the capacity to the initial capacity $C_{0}$ as expressed in Eq. (8) are entered in that figure. Although the phenomena of the stress and strain in this wave propagation test by the shock tube are considerably rapid, it is found that the responsibility of this condensertype strain meter is excellent.

Fig. 5 shows the stress-strain curves which are
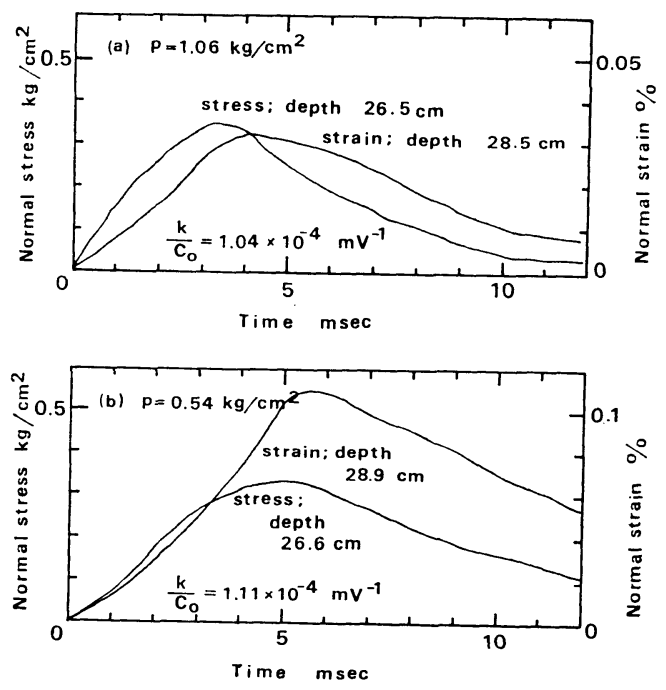

Fig. 4 Typical records of the stress and the strain forms.

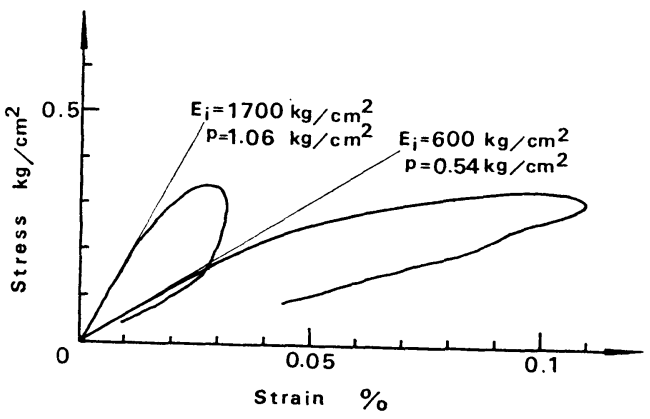

Fig. 5 The stress-strain curves. 
constructed by picking up the stress and the strain values corresponding to the same instant of time in Figs. 4 (a) and (b), respectively. Though each stress-strain curve expresses a large hysteresis loop for a given dynamic stress cycle, the plastic deformation does not seem to occur. Consequently, the physical behavior of the clay sample is considered viscoelastic at the range of a given strain level less than $10^{-3}$. The initial tangent moduli $E_{i}$ obtained from the stress-strain relationship are $1700 \mathrm{~kg} / \mathrm{cm}^{2}$ in the case of $p=1.06 \mathrm{~kg} / \mathrm{cm}^{2}$ and $600 \mathrm{~kg} / \mathrm{cm}^{2}$ for $p=0.54 \mathrm{~kg} / \mathrm{cm}^{2}$, respectively, while the Young's moduli $E$ calculated from rod velocity $c_{R}$ and density $\rho$ by the following equation;

$$
E=\rho c_{R}^{2}
$$

are 1730 and $695 \mathrm{~kg} / \mathrm{cm}^{2}$, respectively. $E_{i}$ and $E$ are almost identical, in both cases and it is con. sidered that this type of strain meter has an enough confidense.

\subsection{Unconfined Compression Test}

A distribution of normal strain in soil sample in unconfined compression test is observed by using this type of soil strain meter. The procedure of the strain meter is same as that described in 5.1.

In order to know the strain distribution in soil sample, needles are in each test embedded in one of upper, middle and lower parts of soil sample as shown in Fig. 6. Fig. 7 shows the relationship between the local strain measured by the soil strain meter and the average strain by dial gage. The soil samples used are relatively soft clay with $5.2 \mathrm{~cm}$ in diameter and about $10 \mathrm{~cm}$ in

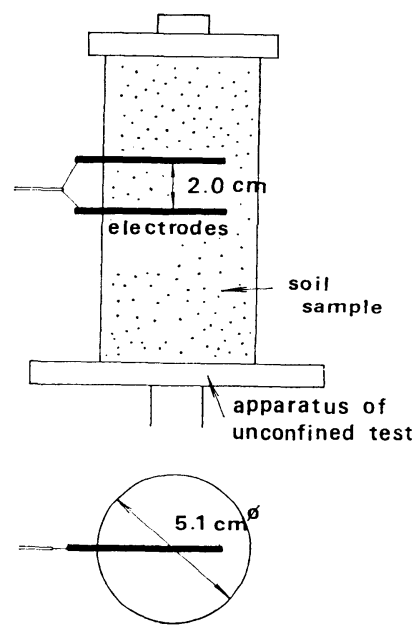

Fig. 6 The manner of embedding needles into the sample of unconfined compression test.

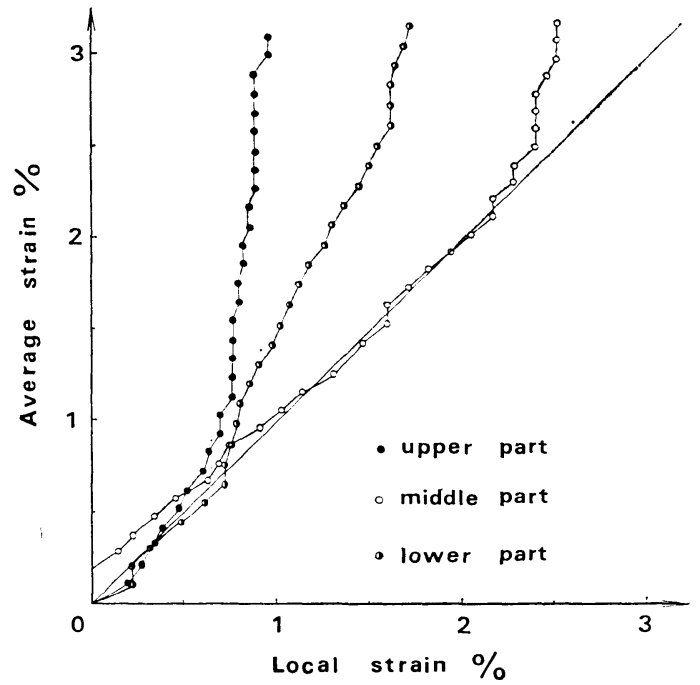

Fig. 7 The relationships between the local strain and the average strain in soft clay samples.

height. Their failure forms are ductile. It is found from this figure that the distribution of normal strain is nearly uniform up to the strain of $0.8 \%$, and non-uniform for much more strain. The local strain in a middle part of sample is same as the average strain up to about $2.5 \%$, and much more compressed, the local strain does not increase and the soil in this part flows in lateral.

Fig. 8 is the results for a relatively stiff clay.

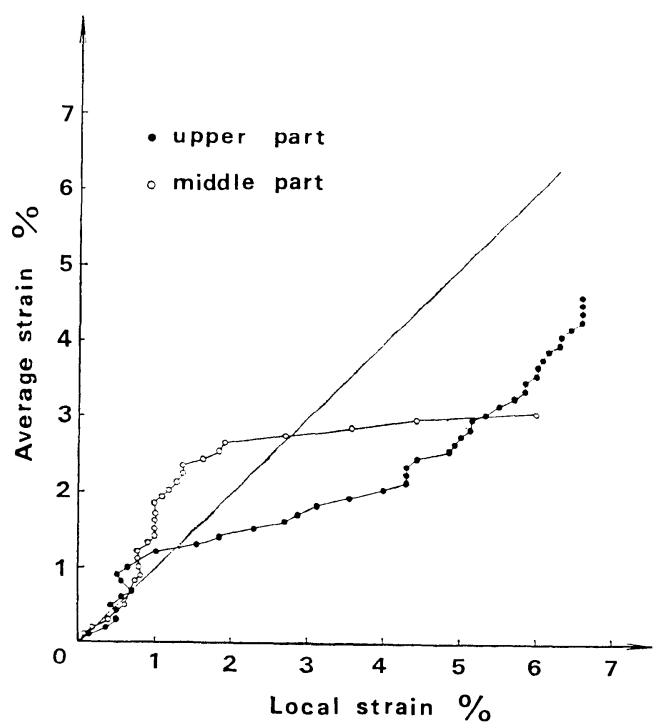

Fig. 8 The relationships between the local strain and the average strain in stiff clay samples. 
Failure form in this case is brittle. The strain distribution is also uniform up to strain $0.8 \%$ same as above described one. Under further compression the local strain in middle part increases gradually and the one in upper part increases rapidly. This is due to that the samples are broken down.

\section{CONCLUSIONS}

It is found through this study that the condensertype infinitesimal displacement meter can be used to measure the one-dimensional normal strain in soil. By using the thin needles as the electrodes of a condenser embedded in soil, the disturbance in soil sample can be decreased and the soil strain can be measured much more accurately. The troublesome calibration test described the previous paper $^{1)}$ is needless and when a pair of needles is embedded in soil, it does not need to read the initial spacing of them, provided the initial capacity $C_{0}$ and the constant $k$ are examined from Eq. (8). Since the responsibility of this meter is excellent even in higher frequency range, it is suitable to measure the dynamic strain in soil.

\section{REFERENCES}

1) Akai, K., M. Hori, N. Ando and T. Shimogami: Shock Tube Study on Stress Wave Propation in Confined Soils, Proc. of JSCE,
No. 200, April, 1972, pp. 127-141.

2) Takenaka, J.: Measurements of Stress, Asakura Shoten, 1964, pp. 261-285 (in Japanese).

3) Truesdale, W. B. and R. B. Schwab: Soil Strain Gage Instrumentation, Proc. Int. Symp. Wave Propagation and Dynamic Properties of Earth Materials, Univ. of New Mexico, 1967, pp. 931-941.

4) Vey, E. and L. V. Strauss: Stress-Strain Relationships in Clay Due to Propagating Stress Waves, Proc. Int. Symp. Wave Propagation and Dynamic Properties of Earth Materials, Univ. of New Mexico, 1967, pp. 575-586.

5) Deroock, B. and A. W. Cooper: Relation between Propagation Velocity of Mechanical Waves through Soil and Soil Strength, Proc. Int. Symp. Wave Propagation and Dynamic Properties of Earth Materials, Univ. of New Mexico, 1967, pp. 905-912.

6) Nishigaki, Y.: Changes of Young's Modulus of Clay with Strain Level, Preprint, 26th Conf. of JSCE, 1971, pp. 93-96 (in Japanese).

7) Hori, M. and F. Oka: On the Measurement of Soil Strain (the second report), Preprint, Conf. of JSCE, Kansai Branch, 1972, III-43 (in Japanese).

8) Shimogami, T.: Study on Stress Propagation through Cohesive Soils by Means of Triaxial Shock-Tube, Master Thesis of Kyoto University, 1973 (in Japanese).

(Received May 10, 1973) 